\title{
Successful Treatment of Chronic Constipation With Functional Electrical Stimulation of the Abdominal Muscles: A Case Report
}

\author{
Christine Singleton ${ }^{\mathrm{a}}$, Abdel Magid Bakheit ${ }^{\mathrm{b}}$
}

\begin{abstract}
Intractable chronic constipation is common in patients with multiple sclerosis and often reduces the quality of life. The drug management of this symptom is usually only partially effective and may cause adverse effects. We report here the successful treatment of severe chronic constipation with functional electrical stimulation in a patient with multiple sclerosis.
\end{abstract}

Keywords: Electrical stimulation; Constipation; Multiple sclerosis

\section{Introduction}

Chronic constipation resistant to medical treatment is common in patients with multiple sclerosis (MS). In a study of 280 unselected patients with MS $43 \%$ of them were found to have severe constipation and the prevalence of this symptom was not influenced by disease severity or the presence of spinal pathology [1].

Severe intractable constipation is often associated with increased morbidity, especially in patients with MS. For example, Dasgupta et al [2] have found that MS patients were 3 to 4 times more likely to be admitted to hospital with faecal impaction and megacolon than patients with other neurological conditions. Furthermore, severe constipation also has a

Manuscript accepted for publication July 5, 2013

${ }^{\mathrm{a} C l i n i c a l}$ Lead for FES Service \& Clinical Specialist-Physiotherapy, West Midlands Rehabilitation Centre, 91 Oak Tree Lane, Birmingham B29 6JA, UK

${ }^{\mathrm{b}}$ Neurological Rehabilitation, Moseley Hall Hospital, Birmingham B13 $8 \mathrm{JL}, \mathrm{UK}$

${ }^{\mathrm{c}}$ Corresponding author: Abdel Magid O. Bakheit, Neurological Rehabilitation, Moseley Hall Hospital, Birmingham B13 8JL, UK.

Email: Magid.bakheit@bhamcommunity.nhs.uk

doi: http://dx.doi.org/10.4021/jmc1381w significant impact on the individual's quality of life and social participation. In a recent survey [3] $47 \%$ of MS patients reported that they were forced to make life style changes and $15 \%$ had to give up paid employment because of the impact of constipation on their normal activities of daily living. Bowel dysfunction may also affect patients' sex life and intimate relationships.

The traditional management of severe constipation with dietary modification, oral laxatives, suppositories, enemas, digital anal stimulation and manual evacuation are usually only partially effective. Severe cases may require surgical treatment, such as subtotal colectomy and ileorectal anatomisis. In recent years sacral nerve stimulation has been shown to reduce constipation in patients with diminished rectal sensation, slow colonic transit time and reduced urge to defecate [4-7]. However, the implantation of the stimulator is an invasive surgical procedure that is associated with potentially serious complications such as infection, cerebrospinal fluid collection around the receiver and receiver failure [8].

We report here a case of successful treatment of severe chronic constipation with non-invasive functional electrical stimulation of the abdominal muscles.

\section{Case Report}

A 61 years old lady with MS which was diagnosed 23 years earlier was initially referred to our services for the management of poor mobility associated with spastic paraparesis. She was treated successfully with functional electrical stimulation (FES) of the ankle dorsiflexors which improved clearance of the ground in the swing phase of the gait cycle and enabled independent transfers from bed-to-chair etc.

As a child she had mild polio but no other significant past medical history. She was taking the following daily medication: Gabapentin $1200 \mathrm{mg}$, Amitrityline $20 \mathrm{mg}$, Fluoxetine 20 mg, Senna 1 - 2 tablets, Movicol 4 sachets and Oxybuta$\operatorname{nin} 2 \mathrm{mg}$.

At a routine clinic review the patient complained of severe constipation of 12 months duration. In addition, she reported abdominal bloating, lack of the urge to defecate 
and absent bowel sensation. She felt her quality of life was reduced because of the restrictions imposed on her by her bowel dysfunction and felt isolated and stressed. Despite treatment with Movicol (4 sachets per day), Senna (1 or 2 nightly) and regular enemas the frequency of her bowel motions was once or twice per week. Her stools consistency was hard and she lacked the urge to defecate. Defecation was time consuming. It required her to strain; and was accompanied by a sense of incomplete evacuation. Her hydration status and diet were adequate. A colonoscopy was normal.

The patient was offered treatment for her constipation with FES. Stimulation of the external oblique and transverse abdominis muscles at $40 \mathrm{~Hz}$ was given using Microstim 2 (Odstock Medical Ltd, Salisbury, Wiltshire, UK, SP2 8BJ). Daily treatment was initially given for 15 minutes and was subsequently increased to 30 minutes. After 4 weeks of continuous use of FES (as well as her usual dose of laxatives) the patient regained the awareness of the urge to defecate and had regular bowel motions with a frequency of 2 to 3 per week and softer stools consistency. However, a few weeks later she developed frequent loose stools. The FES and laxatives were discontinued and she did not have a bowel motion for the following 10 days. She resumed taking movicol and started the FES and had a bowel motion the following day. Since then she continued on the FES regime. The patient reported a newly established pattern of bowel function with normal bowel motions after every three days for two successive days. She reduced her intake of laxatives. She now took 8 sachets of Movicol as required 8 sachets per month instead of 4 per day. She stopped taking Senna and did not require enemas. The abdominal discomfort and bloating reduced. She felt more confident to increase her social engagements because of the improved control of her bowel function. Furthermore, the District Nurse's attendance reduced from one or two visits per month to one every 6 weeks. These benefits were maintained at follow up three months later. There were no adverse effects of treatment. In addition, her core stability improved and was able stand for longer periods and transfer from bed-to-chair etc more easily.

\section{Discussion}

Our patient meets Rome III criteria of functional constipation [9]. Regular treatment with laxatives, enemas and digital evacuation was not very effective. However, her symptoms significantly improved with the regular use of FES. FES was well tolerated and no adverse effects of treatment were reported by the patient or observed by the clinicians. FES of the abdominal muscles has increased the frequency of spontaneous defecation in our patient, reduced the need for laxatives and visits from the district nurse, improved the patient's confidence and reduced the restriction of her style which was imposed by chronic constipation. The patient felt that her quality of life had improved stating that "improved self confidence means I can be my own advocate rather than feeling vulnerable or passive". These results are similar to those reported after implantation of anterior sacral root stimulators [4-8].

Causes of chronic constipation in patients with MS. include slow bowel transit time, weakness of abdominal and pelvic floor muscles, reduced bowel sensation and reduced gastro-colic reflex. Other factors, such as dietary fibre content, dehydration and medication, also contribute to constipation. However, slow bowel transit time in the distal colon appears to be the main factor in MS [10].

The effectiveness of anterior sacral root stimulation in the management of slow transit time chronic constipation in neurological patients appears to be due the simultaneous increase in colorectal contractions and relaxation in the anal sphincter [8, 11, 12]. It reduces constipation by decreasing the colonic transit time [13]. FES may have a similar mechanism of action.

FES enables the contractions of weak muscles and thus improves motor function, e.g. stimulation of the partially paralysed tibialis anterior muscle assists ankle dorsiflexion of the hemiparetic limb in the swing phase of the gait cycle [14]. It is possible that the contractions of the abdominal muscles evoked by FES increase the intra abdominal pressure and/ or the bowel motility and this may account for the increased frequency of bowel evacuation. However, irrespective of the mechanism of action, the advantage of FES over sacral root stimulation is that it is non-invasive, easy to use and safe.

Further research is needed to establish the mechanism of action and the efficacy of FES in the treatment of chronic constipation due to reduced colonic motility and slow faecal colonic transit.

\section{Conflict of Interest}

None declared.

\section{References}

1. Hinds JP, Eidelman BH, Wald A. Prevalence of bowel dysfunction in multiple sclerosis. A population survey. Gastroenterology. 1990;98(6):1538-1542.

2. DasGupta R, Fowler CJ. Bladder, bowel and sexual dysfunction in multiple sclerosis: management strategies. Drugs. 2003;63(2):153-166.

3. Norton C, Chelvanayagam S. Bowel problems and coping strategies in people with multiple sclerosis. $\mathrm{Br} \mathrm{J}$ Nurs. 2010;19(4):220, 221-226.

4. Chang HS, Myung SJ, Yang SK, Yoon IJ, Kwon OR, Jung HY, Hong WS, et al. Functional constipation with 
impaired rectal sensation improved by electrical stimulation therapy: report of a case. Dis Colon Rectum. 2004;47(6):933-936.

5. Chang HS, Myung SJ, Yang SK, Jung HY, Kim TH, Yoon IJ, Kwon OR, et al. Effect of electrical stimulation in constipated patients with impaired rectal sensation. Int J Colorectal Dis. 2003;18(5):433-438.

6. Chiarioni G, Chistolini F, Menegotti M, Salandini L, Vantini I, Morelli A, Bassotti G. Short-term effects of magnetic sacral dermatome stimulation for idiopathic slow transit constipation: sham-controlled, cross-over pilot study. Diseases of the Colon and Rectum. 2004; 47: 346-353.

7. Binnie NR, Smith AN, Creasey GH, Edmond P. Constipation associated with chronic spinal cord injury: the effect of pelvic parasympathetic stimulation by the Brindley stimulator. Paraplegia. 1991;29(7):463-469.

8. MacDonagh RP, Sun WM, Smallwood R, Forster D, Read NW. Control of defecation in patients with spinal injuries by stimulation of sacral anterior nerve roots. BMJ. 1990;300(6738):1494-1497.

9. Longstreth GF, Thompson WG, Chey WD, Houghton
LA, Mearin F, Spiller RC. Functional bowel disorders. Gastroenterology. 2006;130(5):1480-1491.

10. Waldron DJ, Horgan PG, Patel FR, Maguire R, Given HF. Multiple sclerosis: assessment of colonic and anorectal function in the presence of faecal incontinence. Int J Colorectal Dis. 1993;8(4):220-224.

11. Varma JS. Autonomic influences on colorectal motility and pelvic surgery. World J Surg. 1992;16(5):811-819.

12. Valles M, Rodriguez A, Borau A, Mearin F. Effect of sacral anterior root stimulator on bowel dysfunction in patients with spinal cord injury. Dis Colon Rectum. 2009;52(5):986-992.

13. Clarke MC, Chase JW, Gibb S, Robertson VJ, CattoSmith A, Hutson JM, Southwell BR. Decreased colonic transit time after transcutaneous interferential electrical stimulation in children with slow transit constipation. J Pediatr Surg. 2009;44(2):408-412.

14. Burridge JH, McLellan DL. Relation between abnormal patterns of muscle activation and response to common peroneal nerve stimulation in hemiplegia. Journal of Neurology Neurosurgery and Psychiatry 2000; 69: 353361. 